\title{
On the Correlation of Solar Activity and Troposphere on the GNSS/EGNOS Integrity. Fuzzy Logic Approach
}

\author{
Karolina Krzykowska-Piotrowska ${ }^{1, *(\mathbb{D}, \text { Ewa Dudek }}{ }^{1}$, Paweł Wielgosz ${ }^{2}{ }^{\oplus}$, Beata Milanowska ${ }^{2}$ \\ and Jordi Mongay Batalla ${ }^{3}$ (D) \\ 1 Faculty of Transport, Warsaw University of Technology, Koszykowa 75, 00-662 Warsaw, Poland; \\ ewa.dudek@pw.edu.pl \\ 2 Faculty of Geoengineering, University of Warmia and Mazury in Olsztyn, Oczapowskiego 2, \\ 10-719 Olsztyn, Poland; pawel.wielgosz@uwm.edu.pl (P.W.); beata.milanowska@uwm.edu.pl (B.M.) \\ 3 Institute of Telecommunications, Warsaw University of Technology, Nowowiejska 15/19, \\ 00-665 Warsaw, Poland; jordi.mongay.batalla@pw.edu.pl \\ * Correspondence: karolina.krzykowska@pw.edu.pl
}

check for updates

Citation: Krzykowska-Piotrowska, K.; Dudek, E.; Wielgosz, P.; Milanowska, B.; Batalla, J.M. On the Correlation of Solar Activity and Troposphere on the GNSS/EGNOS Integrity. Fuzzy Logic Approach. Energies 2021, 14, 4534. https://doi.org/10.3390/en14154534

Academic Editors: Juan Luis Bosch Saldaña, Silvio Simani and Philippe Leclère

Received: 31 March 2021

Accepted: 23 July 2021

Published: 27 July 2021

Publisher's Note: MDPI stays neutral with regard to jurisdictional claims in published maps and institutional affiliations.

Copyright: (c) 2021 by the authors. Licensee MDPI, Basel, Switzerland. This article is an open access article distributed under the terms and conditions of the Creative Commons Attribution (CC BY) license (https:/ / creativecommons.org/licenses/by/ $4.0 /)$.

\begin{abstract}
There is a noticeable number of studies carried out on both the operational parameters of Global Navigation Satellite System (GNSS) and the satellite signal itself. Researchers look for, inter alia, proven sources of errors affecting the integrity of the satellite signal because this parameter determines the approval of the system's operational use. It also seems of key importance that the atmospheric conditions, in any area of satellite signal usage, should not be underestimated due to their extensive impact. As the ionospheric refraction seriously limits the operational use of the satellite navigation signal, in this article, the authors attempted to quantify the effect of solar activity (expressed by sunspots) on the signal integrity using fuzzy logic. Fuzzy reasoning is used when information is inaccurate or incomplete and necessitates making decisions under conditions of uncertainty. Thanks to fuzzy sets, there are no obstacles to characterize the degree of intensity of a given phenomenon. In order to look at the problem more broadly, attention was also paid to the tropospheric conditions, and it was verified whether, against the background of cloudiness, precipitation, humidity, pressure and temperature, solar activity affects the integrity to the greatest extent. The integrity measurements from the EGNOS system (PRN120 and PRN126) collected at the monitoring station in Warsaw, Poland in 2014 were used.
\end{abstract}

Keywords: GNSS; satellite navigation signal; solar activity; integrity

\section{Introduction}

An interest in satellite systems applicable to various engineering fields, especially to navigation, has been observed for a long time. Indeed, navigation systems based on GNSS (Global Navigation Satellite System) have their practical application in many areas, e.g., geodesy, transport, and robotics. It may be written that these systems have an unparalleled advantage in navigational technologies due to their high-precision delivery of location in terms of position, time, and velocity on any object or person all over the world. An example of an exemplification of this feature may be Real-Time Precise Point Positioning (RT-PPP) since it can provide centimeter-to-decimeter-level positioning accuracy by using a single receiver anywhere in the world [1]. The wide application of satellite navigation can be found in transport, where it can be used both as object (vehicle) navigation, but also with toll-collection systems on highways, parking-space monitoring systems, or as a vehicle fleet-management subsystem $[2,3]$. The sources of satellite navigation application should be sought in maritime transport, in which GNSS applications have been supporting for years the Automatic Identification System (AIS) [4].

However, it turned out that the use of a satellite navigation system entails the need to correct positioning errors caused by the atmosphere or by signal reflection [5]. The article 
by Zhu et al. [6] proposed eliminating GNSS multipath errors using an integrated measurement system and artificial neural networks. Another source proposes a new method of improving positioning performance measured by pseudoranges in a degraded signal environment [7]. Importantly, the published materials also include researches related to the influence of external factors on the quality of the satellite signal. The team of J.R. Rodriguez Perez uses artificial neural networks to analyze the accuracy of measurements made with the GNSS technology when the GPS receiver is located in a forested environment [8]. Considerable attention is paid to the influence of the ionosphere on GNSS [9-11], and the ionosphere is being treated as one of the main error sources in positioning and navigation; thus, information about the ionosphere is mandatory for precise GNSS applications [12-14]

Indeed, the issue of the development and implementation of satellite systems has gained the attention of numerous scientific communities and providers of navigation services in recent years. This technology shows the innovative potential that meets the increasing needs of transport users, robots and others. According to [15], by 2025, service providers will have the largest share of revenues from the sale of GNSS applications in General Aviation and business air transport. These assumptions meet the concept of the SESAR (Single European Sky ATM Research) program to introduce the so-called flexible airspace, eliminating restrictive flight plans and introducing $4 \mathrm{D}$ trajectories in air traffic. In view of the indicated plans and assumptions-it is justified to conduct research on predicting the value of the signal integrity and, thus, facilitate the decision to use it through navigation. At the same time, it is worth emphasizing that the EU Agency for the Space Programme (EUSPA) constantly observes the EGNOS signal, and although its performance in terms of parameters is improving, the ionospheric factor was indicated as one of the main causes of interference [16].

Around satellite navigation systems, an important issue of signal integrity has also been raised in the literature [3,17]. Bijjahalli et al. [18] presented an integrity augmentation system that can detect GNSS error sources and alert the navigation system of an autonomous ground vehicle in a timely manner. The system is developed by modeling GNSS error sources, such as antenna masking, signal attenuation, and multipath, and assigning threshold values for generating integrity alerts. Integrity may be defined as a measure of the probability that can be assigned to the correctness of the information provided by the navigation system. Usually, in augmentation satellite systems, such as the European Geostationary Navigation Overlay Service (EGNOS), it is reflected in the Protection levels (PL) and Position errors (PE) values observed during system operation [19]. System integrity monitoring is possible by RAIM (Receiver Autonomous Integrity Monitoring) technique, which analyzes the correctness of information received from satellites. According to literature sources [20,21], SAIM (Satellite Autonomous Integrity Monitoring) technique will become an alternative to RAIM in the future. State-of-the-art shows that there are several trends in this area, i.e.,

1. striving to minimize, and if possible eliminate, errors in the propagation of the satellite signal [13];

2. searching for new solutions for satellite signal integrity monitoring (RAIM, SAIM) [20,21];

3. studying the influence of atmospheric conditions on the satellite signal $[16,22,23]$.

Not surprisingly, model analysis is predominantly used in these trends, and also nowadays, artificial neural networks are used.

In this article, the authors attempt to quantify the effect of solar activity (expressed by sunspots) on satellite navigation signal integrity, using fuzzy logic. However, in order to look at the problem more broadly, attention was also paid to other atmospheric (tropospheric) conditions, and in this case, unlike other research in the literature [24-27], it was verified whether, against the background of cloudiness, precipitation, humidity, pressure and temperature, solar activity affects the integrity to the greatest extent. Moreover, research usually deals with accuracy rather than signal integrity. For this purpose, the integrity measurements from the EGNOS (PRN120 and PRN126) system collected at the monitoring station in Warsaw, Poland, in 2014 were used. The research contribution of 
this article focuses on several aspects. Firstly, studies of the effects of the solar activity on satellite signal integrity were conducted. Secondly, the use of a fuzzy approach was considered. Thirdly, result verification with a statistical analysis that shows the direct correlations between variables was undertaken. Finally, tropospheric factors were added to the research, including not only humidity, pressure and temperature, but also cloudiness and precipitation, which is not found in the traditional approach. The article has the following structure: introduction and review of state-of-the-art research (1), wider presentation of the research problem (2), materials and method devoted to fuzzy logic (3), results and discussion (4), verification (5), conclusions (6), literature.

\section{Research Problem}

The operational limitation of satellite systems usage can be caused by various errors that are inherent in the operation of these systems. In general, errors in satellite systems can be divided into four groups: signal propagation errors (ionospheric errors, tropospheric errors, multipath errors); relativistic errors; system operation errors (satellite ephemeris errors, satellite clock errors); receiver errors, including DOP (Dilution of Precision) [1].

On the way from the satellite to the receiver antenna, the satellite signal passes through the atmosphere, which affects the signal velocity. From the radio wave propagation perspective, the Earth's atmosphere can be divided into the neutral part-troposphere, and the ionized part-ionosphere. The state of the ionosphere, in the context of satellite systems, is determined by Total Electron Content (TEC): the total number of electrons concentrated between a satellite and a receiver along a column with a cross-section equal to $1 \mathrm{~m}^{2}$. TEC is directly related to the ionospheric delay of satellite signals (1). The delay results from a change in the group and phase velocity of the wave and depends on the frequency of the signal. Since the ionosphere is a dispersive medium for GNSS frequencies, the ranging codes are delayed and the carrier phases are advanced [28,29].

$$
\Delta \text { Ion }=\frac{40.3}{f^{2}} \text { TEC }
$$

where:

$\Delta$ Ion is the GNSS signal delay;

$f$ is the signal frequency.

The TEC level is primarily driven by the solar radiation, mainly ultraviolet and $\mathrm{x}$-rays. Consequently, the state of the ionosphere is driven by solar activity, which is manifested by changes on the surface of the sun and its atmosphere. The level of solar activity is related to the changes in the distribution and number of sunspots (SSN). A sunspot is a darker area on sun surface, which is a source of increased electromagnetic radiation. The solar sunspot cycle is approximately 11 years, and in one cycle, the sun changes its activity from a minimum to the next minimum [30]—see Figure 1.

The study of TEC, its modeling and prediction has become a popular problem in modern science in the context of progress in satellite technology. Due to the proven significant influence of TEC on the quality of the received satellite signal, much attention has been focused on TEC prediction, e.g., by using artificial neural networks [31-34]. Another important field of study is radio occultation technique, which is used both for studying ionosphere, space weather, and neutral atmosphere $[35,36]$. 


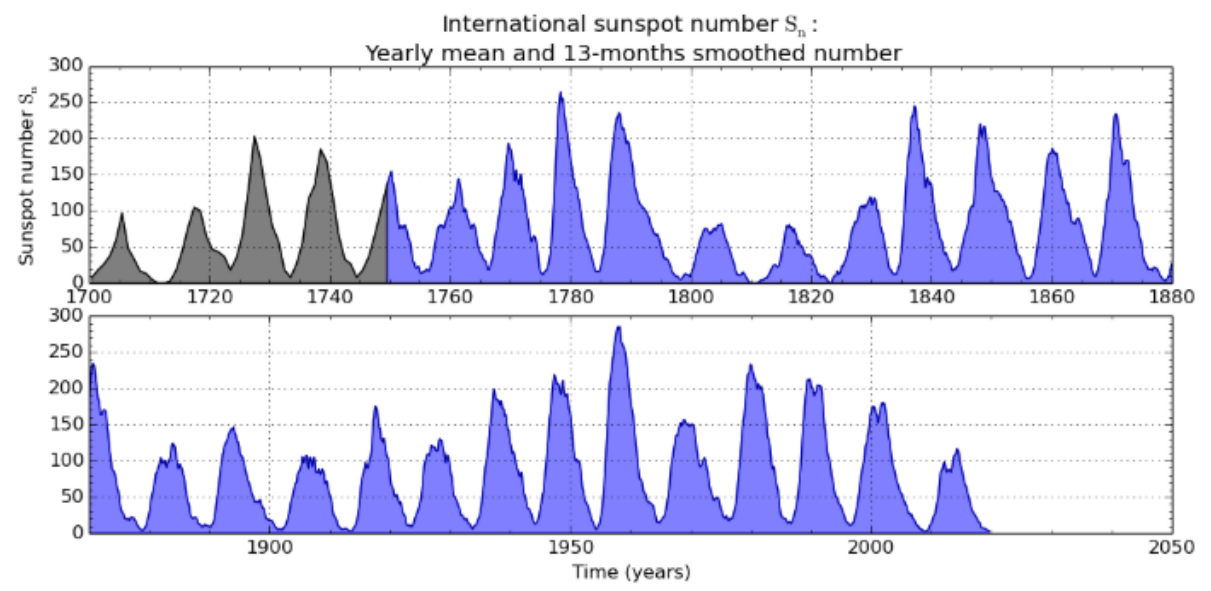

Figure 1. Observation of SSN over the years in cycles [37].

The troposphere, on the other hand, is the lowest and the thinnest layer of the atmosphere. It extends from the Earth's surface to a height of about $40 \mathrm{~km}$ [1]. All the most important phenomena that shape the weather and climate on Earth take place in the troposphere. For GNSS frequencies the troposphere is a non-dispersive medium, so both code measurements and carrier phases are delayed, regardless of the signal frequency. Tropospheric error is caused by both the dry and wet parts of the troposphere. The dry (hydrostatic) part consists of atmospheric pressure, air density and air temperature (2). The wet (non-hydrostatic) part of the troposphere is related to the water vapor content (3). This content is due to weather patterns in the troposphere, for example, the presence of clouds and the precipitation introduce almost $99 \%$ of moisture in the lowest part of the atmosphere. The water vapor content, thus, also depends on altitude and atmospheric phenomena (storms, fronts) [38].

$$
\begin{gathered}
\Delta_{d}^{\text {Trop }}(E)=\frac{10^{-0}}{5} \frac{77.64}{\sin \sqrt{E^{2}+6.25}} \frac{p}{T}[40136+148.72(T-273.16)] \\
\Delta_{w}^{\text {Trop }}(E)=\frac{10^{-6}}{5} \frac{\left(-12.96 T+3.718 \cdot 10^{5}\right)}{\sin \sqrt{E^{2}+2.25}} \frac{e}{T^{2}} 11,000
\end{gathered}
$$

where:

$\Delta_{d}^{\text {Trop }}$ and $\Delta_{w}^{\text {Trop }}$ is the tropospheric delay for the dry and wet component, respectively; $E$ is the elevation angle at the observing site;

$p$ is the atmospheric pressure;

$T$ is the temperature;

$e$ is the partial pressure of water vapor.

While integrity-according to the International Civil Aviation Organization (ICAO), is defined as a measure of confidence in the correctness of information provided by the system, it includes the ability of the system to deliver appropriate warnings (alerts) to the user at a predetermined time, which contain information on when the system should not be used. In the context of integrity modeling, in order to determine if the position error is acceptable, an alert limit is set so that it is possible to reflect the maximum allowable position error that will not jeopardize the ongoing operation using a satellite signal (e.g., aircraft take-off). It should be remembered that navigation using satellite systems, and thereby, the signal, is provided simultaneously to many objects over a large area-often one or several continents. The impact of the satellite system integrity loss could be much greater than with conventional navigation methods. Sufficiently, information provided about the loss of signal integrity should result in the resignation from the use of satellite navigation or the cessation of operations (e.g., take-off or landing of an aircraft, moves of ship or robots). Moreover, it is an individual and unique feature of satellite navigation 
to adapt the navigation capability to the changing constellation of satellites. The impact of changes in the space segment may be increased by an additional fault in the ground segment, for example, damage of one of the components [39].

GNSS augmentation systems are also susceptible to the influence of atmospheric effects. Rapid changes in the ionosphere influence signal delays that cannot be corrected in real time. Such cases are rare, and the likelihood of their occurrence varies by region, but cannot be excluded. As a result, errors that arise may distort information and should be taken into account at the system design stage. Determining the requirements for the parameters of the satellite signal and the implementation of augmentation systems are the key processes for introducing satellite navigation $[40,41]$. In Europe, the turning point in this activity was the launch of the SBAS-EGNOS (Satellite Based Augmentation SystemGeostationary Navigation Overlay Service) system [15,42]. It also reaching the milestone in Poland in implementing aviation procedures based on GNSS navigation.

The EGNOS system is a European GPS and GLONASS augmentation system in the fields of air, maritime and road transport. It was designed by the ETG (European Tripartite Group) group, which includes: the European Space Agency ESA, the European Commission EC and the European Organization for the Safety of Air Navigation EUROCON-TROL. The aim of EGNOS is to monitor the integrity of GPS and GLONASS and to increase their accuracy by introducing data corrections. The principle of operation is based on the reception of GNSS signals by ground reference stations [19]. The EGNOS terrestrial segment includes, among others, RIMS (Ranging and Integrity Monitoring Stations) stations. RIMS can be analyzed in terms of the channels constituting them (A, B, and $C)$. Data from channel A is used to calculate data needed for EGNOS messages. Data from the B channel is used to verify the message calculated from the A channel. Data from the C channel is to detect errors in the signal provided by the GNSS satellites. The task of RIMS is to collect data from GNSS satellites and verify them. By dividing RIMS stations into three channels, the EGNOS data waveform is presented in two separate but related cycles, which, according to the minimum fault warning requirements, should take a total duration of no more than $6 \mathrm{~s}$. The first cycle is the processing cycle. It uses only channel A. The course of this cycle consists of measuring the pseudorange to the satellites through the antennas and receivers of the A channel of the RIMS station, then transmission of the received data to the MCC (Mission Control Center) cell: CPF (Central Processing Unit), then generating corrections, integrity information and the entire navigation message that is sent by the NLES (Navigation Land Earth Stations) stations to the EGNOS geostationary satellites. The second cycle is the checking one. The B and C channels are used in here. Data collected by RIMS antennas and receivers from EGNOS satellites and other navigation satellite systems are sent to the MCC-CPF cell. In the CPF, these data are checked and verified for accuracy, correctness, truthfulness and integrity of information. In case of detection of an error in emitted information, the so-called alarm flag informing system recipients of an error or failure is sent.

Integrity for EGNOS is presented in the form of a Stanford diagram. The diagram shows boundaries of the relationship between PE (Position Error), PL (Protection Level) and AL (Alert Limit). The PE level should always be lower than AL. A favorable relationship for integrity also occurs when PE $<$ PL. It can, therefore, be concluded that data integrity will be ensured when $\mathrm{PE}<\mathrm{PL}<\mathrm{AL}$. The relationship $\mathrm{PL}<\mathrm{PE}<\mathrm{AL}$ may also be considered relatively positive, however, when PL $<$ PE, then information distortions may occur $[19,43]$. The distribution of the Stanford diagram is shown in Figure 2.

Owing to the measurements of the EGNOS satellite system signal carried out by, among others, the Polish Air Navigation Services Agency, it is possible to analyze this signal on the basis of its daily parameters. The agency uses a specialized receiver (Septentrio PolaRx3) and appropriate software (PEGASUS 4.7.5.) that can decode the EGNOS data. PEGASUS, developed for research purposes, determines the difference between the position identified by the EGNOS system and the known position of the EGNOS receiver. It is then possible to obtain a positioning error and a number of other important quantities. 
This software was developed to test the performance of SBAS and GBAS (Ground Based Augmentation System) systems in the first place. For each of the geostationary satellites, PEGASUS automatically generates a report once a day. The integrity is interpreted by the number of misleading information (MI) and the horizontal (HPL-Horizontal Protection Level and HPE-Horizontal Position Error) and vertical (VPL-Vertical Protection Level and VPE-Vertical Position Error) statistics in terms of HPL and VPL standard deviations (std), mean values, and 50\%, 95\% and 99\% significance levels [2]. Graphical visualization of integrity is presented via Stanford diagrams.

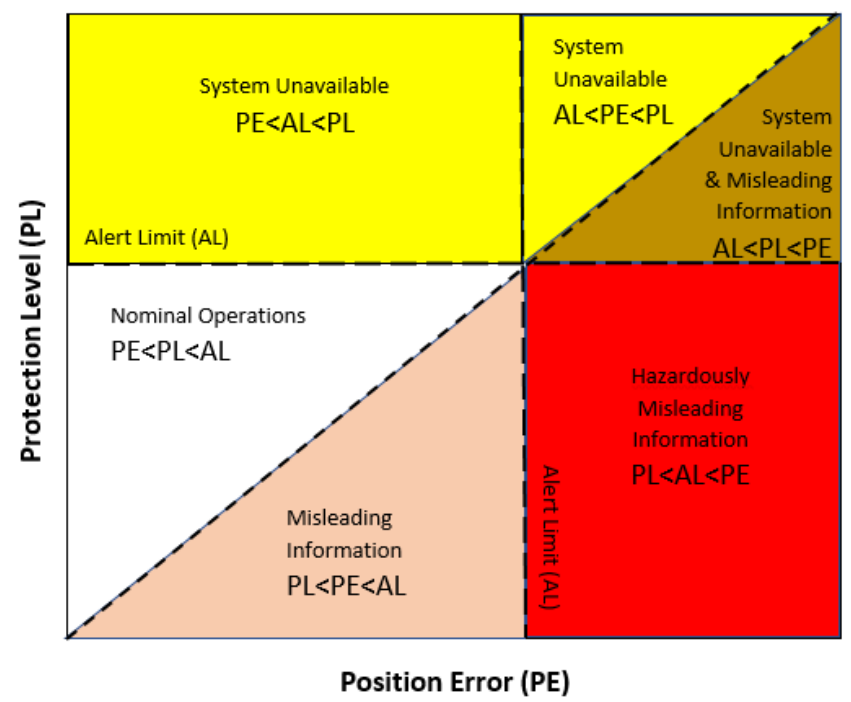

Figure 2. Stanford diagram distribution for EGNOS (Own figure based on [44]).

The analysis and interpretation of PEGASUS reports are particularly important for the purposes of this article. As a result of the agreement and official cooperation with the Polish Air Navigation Services Agency in the conducted research, the data from 2014 generated for the Warsaw monitoring station for the EGNOS PRN120 and PRN126 satellites operating in this period were used. Note that the last solar maximum took place in 2014.

\section{Materials and Methods}

The analysis of phenomena related to the satellite signal is not possible without model analysis. Experimenting on the actual operation can lead to dramatic consequences. Therefore, numerous attempts are made to study the signal and the processes taking place in satellite systems during operation, which have a potential impact on its disturbance.

Fuzzy sets are a suitable tool for assessing the influence of solar activity on the integrity of a satellite signal. A fuzzy set is a mathematical object with a defined membership function that takes values from the range [0,1]. The fuzzy set theory was introduced by Lotfi A. Zadeh in 1965 as an extension of the classical set theory [45]. This reasoning is used when information is inaccurate or incomplete, which necessitates a decision under uncertainty. Knowledge about the phenomenon is subjective and is expressed through the opinion or descriptive research of an expert. It is informally referred to as linguistic uncertainty. Their big advantage is the possibility of graphical presentation of research results, modeling uncertainty and examining the influence of many factors on the phenomenon. There are numerous references to the use of fuzzy sets in the literature for navigation, e.g., [46-48]. For the sake of order, the process of building a linguistic fuzzy model is presented below. Consequently, a universal, simplified course of action was created, based on the available literature on the subject [45,49-51], enabling the construction of a comprehensive fuzzy model. In the process of its building, it is possible to distinguish several stages. 
Stage 1. Definition of a fuzzy set. Fuzzy set $A$ in the space of considerations $X=\{x\}$, meaning set $A$ in $X$ is defined as a set of pairs:

$$
A=\left\{\left(\mu_{A}(x), x\right)\right\}
$$

where:

$\mu_{A}: X \rightarrow[0,1]$ is a fuzzy set membership function;

$\mu_{A}(x) \in[0,1]$ is the degree of belonging of an element $x \in X$ in fuzzy set $A$.

The membership function $\mu_{A}: X \rightarrow[0,1]$ to each element $x \in X$ assigns its degree of belonging to the fuzzy set $A$. Three cases of belonging can be distinguished:

(a) $\quad \mu_{A}(x)=1$-full belonging of element $x$ to fuzzy set $A, x \in A$;

(b) $\mu_{A}(x)=0$-no belonging of element $x$ to fuzzy set $A, x \notin A$;

(c) $0<\mu_{A}(x)<1$-partial belonging of element $x$ to fuzzy set $A$.

Stage 2. Fuzzification. The selection of the membership function shape takes place before defining the linguistic area of considerations. The mathematical notation of Gauss function is presented below (5). Gaussian function is one of the commonly used membership functions where $\bar{x}$ is the center and $\sigma$ is the width of the Gaussian curve. Also, this curve was chosen for researches carried out in this paper.

$$
\mu_{A}(x)=\exp \left(-\frac{1}{2}\left(\frac{x-\bar{x}}{\sigma}\right)^{2}\right)
$$

In Table 1, the first two stages: fuzzy set definition, determination of linguistic variables and membership function identification are presented. Table 1 refers to the research problem presented in this paper where inputs are identified by sunspots, number of daily sunspot observations and standard deviation of daily sunspot number. The outputs are identified by integrity of PRN120 and PRN126. Even though we do not expect the number of sunspot observations and related standards deviation to have any impact on the signal integrity, we added these parameters to test/validate our approach.

\begin{tabular}{|c|c|c|c|c|c|}
\hline $\begin{array}{l}\text { Input/ } \\
\text { Output }\end{array}$ & Linguistic Variable & Measure & $\begin{array}{l}\text { The Range of } \\
\text { Measurement Values } \\
\text { from a Given Period }\end{array}$ & $\begin{array}{l}\text { Shape of the } \\
\text { Membership Function }\end{array}$ & Linguistic Values \\
\hline \multirow{3}{*}{$x_{1}$} & \multirow{3}{*}{ SSN } & \multirow{3}{*}{ Number of sunspots } & \multirow{3}{*}{$40-220$} & \multirow{3}{*}{ Gauss curve } & small \\
\hline & & & & & medium \\
\hline & & & & & large \\
\hline \multirow{3}{*}{$x_{2}$} & \multirow{3}{*}{$\begin{array}{l}\text { SSN standard } \\
\text { deviation }\end{array}$} & \multirow{3}{*}{$\begin{array}{c}\text { Value of standard } \\
\text { deviation }\end{array}$} & \multirow{3}{*}{$3.0-16.5$} & \multirow{3}{*}{ Gauss curve } & small \\
\hline & & & & & medium \\
\hline & & & & & large \\
\hline \multirow{3}{*}{$x_{3}$} & \multirow{3}{*}{$\begin{array}{l}\text { Daily number of } \\
\text { SSN observations }\end{array}$} & \multirow{3}{*}{$\begin{array}{l}\text { Number of } \\
\text { observations }\end{array}$} & \multirow{3}{*}{$6-21$} & \multirow{3}{*}{ Gauss curve } & small \\
\hline & & & & & medium \\
\hline & & & & & large \\
\hline \multirow{3}{*}{$y_{1}$} & \multirow{3}{*}{ Integrity of PRN120 } & \multirow{3}{*}{ PL-PE ${ }^{1}$} & \multirow{3}{*}{$9.54-12.16$} & \multirow{3}{*}{ Gauss curve } & low \\
\hline & & & & & moderate \\
\hline & & & & & high \\
\hline \multirow{3}{*}{$y_{2}$} & \multirow{3}{*}{ Integrity of PRN126 } & \multirow{3}{*}{$\mathrm{PL}_{-\mathrm{PE}}{ }^{1}$} & \multirow{3}{*}{$9.55-15.875$} & \multirow{3}{*}{ Gauss curve } & low \\
\hline & & & & & moderate \\
\hline & & & & & high \\
\hline
\end{tabular}

Table 1. Definition of the fuzzy sets including membership functions.

${ }^{1}$ PL—Protection Level, PE—Position Error. The higher difference between PL and PE, the higher integrity of the satellite signal. This measure is dedicated to EGNOS, according to [39]. 
A shape of membership function and the range of measurement values for input variables $x_{1}, x_{2}, x_{3}$ and output variables $y_{1}, y_{2}$ are shown through MATLAB Fuzzy Logic Toolbox, according to Table 1 in the Figures 3 and 4.

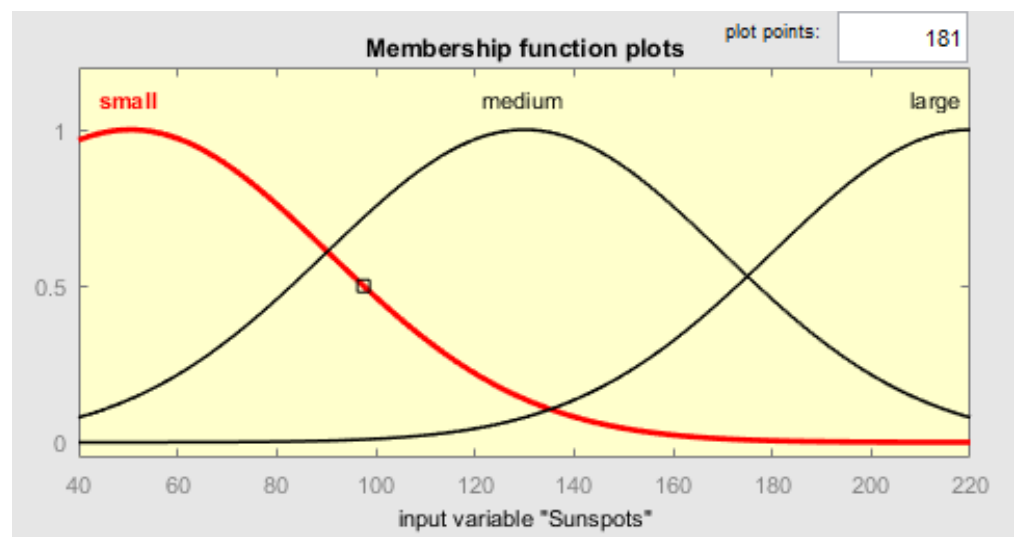

(a)

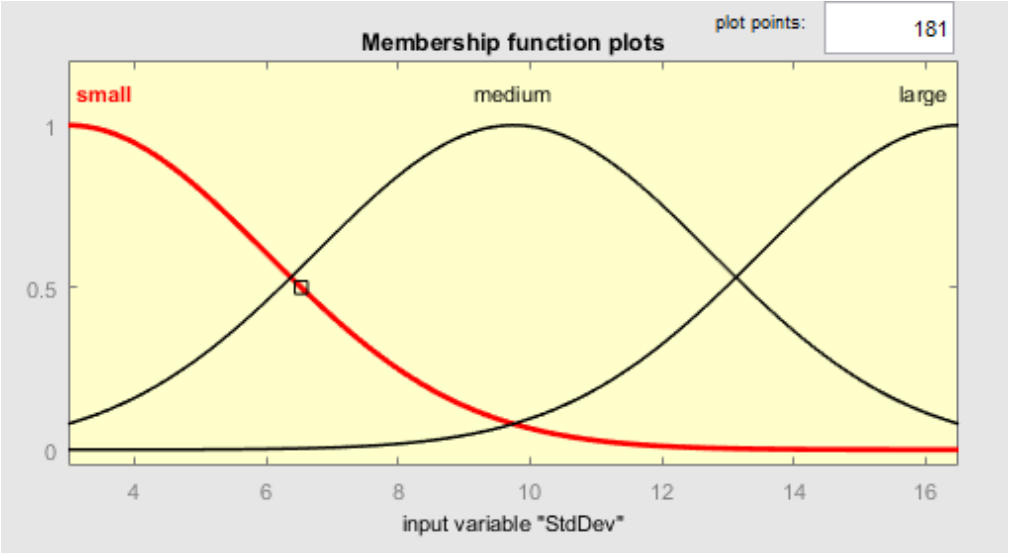

(b)

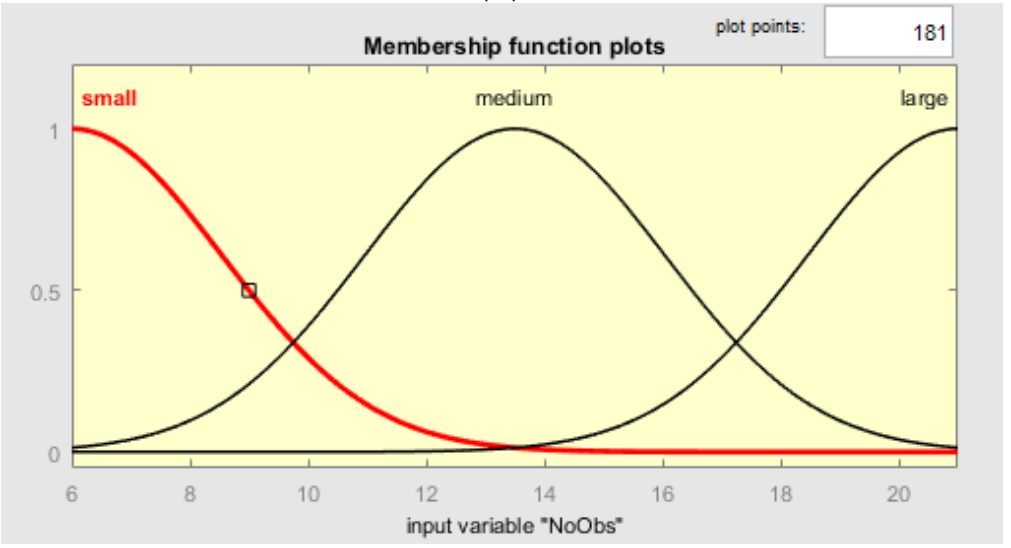

(c)

Figure 3. The shape of the membership function for input variables $x_{1}(\mathbf{a}), x_{2}(\mathbf{b}), x_{3}(\mathbf{c})$ (Own study in MATLAB Fuzzy Logic Toolbox).

Each curve represents the degree of belonging of a given linguistic variable to a function. The $x$-axis (horizontal) specifies a range of values for each variable, e.g., the number of sunspots ranges from 40 to 220 . The $y$-axis values, in turn, range from 0 to 1 and represent the severity of the phenomenon, e.g., the value of 100 sunspots is small with probability 0.5 , medium with probability 0.7 , and large with probability 0 . 


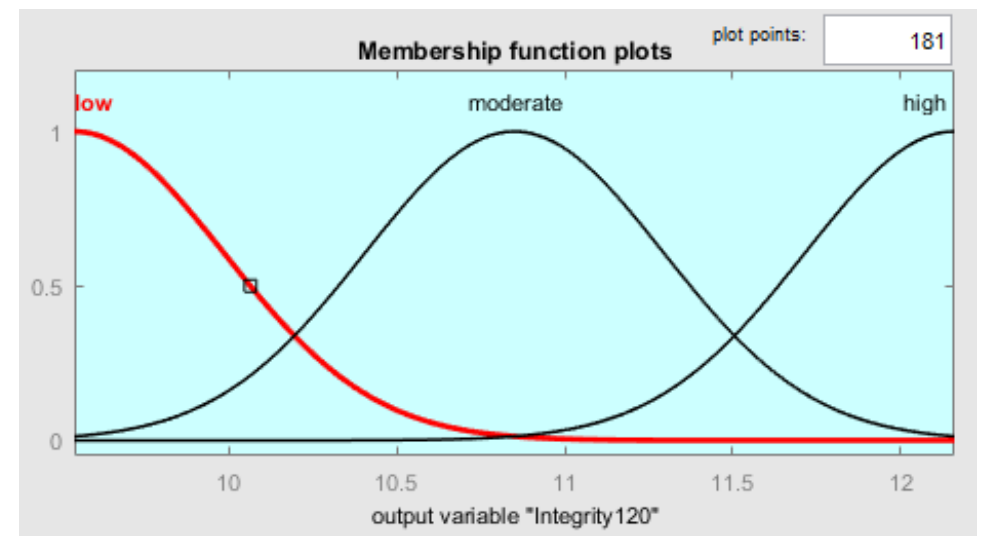

(a)

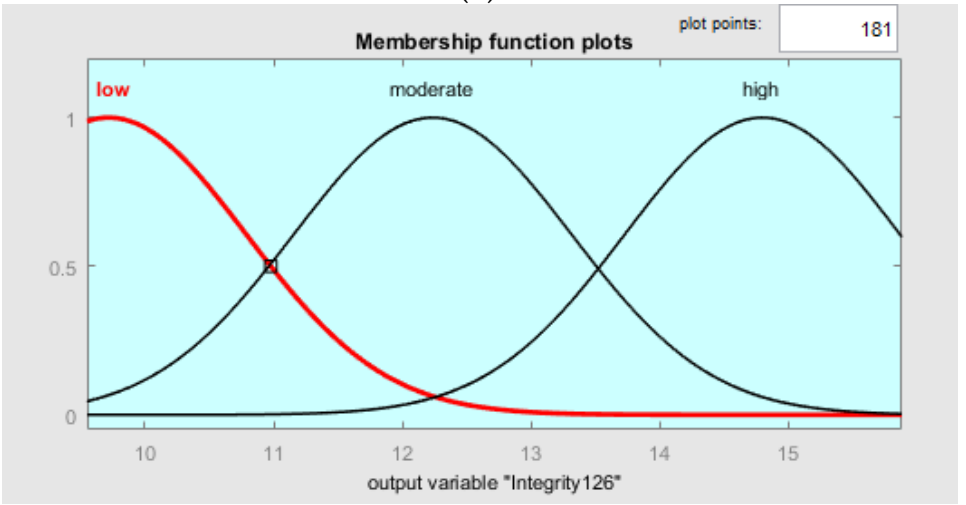

(b)

Figure 4. The shape of the membership function for output variables $y_{1}(\mathbf{a}), y_{2}$ (b) (Own study in MATLAB Fuzzy Logic Toolbox).

Stage 3. Inference. The inference is served by the base of rules, which is created after the process of assigning the membership function to linguistic variables. Two types of architectures can be used in this step: Mamdani or Takagi-Sugeno [48,49]. The architecture of Mamdani was chosen in this research. Rules may be written as follows:

$$
\begin{aligned}
& \text { IF } x=P M A N D y=P B \text { THEN } \Delta u=P B \\
& \text { IF } x=P B \text { AND } y=P B \text { THEN } \Delta u=P B
\end{aligned}
$$

where:

$x, y$-linguistic variables;

$P M, P B$-values of linguistic variables (low, moderate, high);

$\Delta u$-control variable (output variable).

There are different sources of creating the base of rules. Among them, the following may be distinguished: knowledge obtained from experts, scientific experience, literature sources, other research tools. The rule base, according to the selected architecture, is used for inference, which consists of determining the fuzzy value of the output variable, knowing the set of rules representing the relationships between the input and output variables and some fuzzy values of the input variables.

Stage 4. Defuzzification. Having obtained a fuzzy value of the output variable, it should be defuzzified, that is, the corresponding strict (non-fuzzy) value should be found. Below, a mathematical formula representing a center of gravity method is presented (7). It is one of the most commonly used for defuzzification process. It is assumed that there 
is a fuzzy set $A$ identified in $X=\left\{x_{1}, x_{2}, \ldots, x_{n}\right\}$. A non-fuzzy value is searched for $a \in\left[x_{1}, x_{n}\right]$, which best represents $A$.

$$
a=\frac{\sum_{i=1}^{n} x_{i} \mu_{A}\left(x_{i}\right)}{\sum_{i=1}^{n} \mu_{A}\left(x_{i}\right)}
$$

Shall it be assumed that a non-fuzzy value is $a \in X=\left\{x_{1}, \ldots, x_{n}\right\}$, for which $\mu_{A}(x)$ takes the maximum value.

Building a model and conducting research is subject to appropriate data. In order to build the fuzzy model, measurements from year 2014 and the Warsaw station for PRN120 and PRN 126 satellites were used together with data on solar activity, including the number of sunspots, the number of observations, and SSN standard deviation from The Space Research Center of the Polish Academy of Sciences. In total, 181 rules of inference were distinguished.

\section{Results and Discussion}

As an initial result of the conducted research, it is interesting to see that there is some coincidence between the number of the SSN observations and the integrity of the satellite signal. In case of both, the PRN120 and PRN126 satellites, a large number of SSN observations correlate positively with the signal integrity (Figure 5).

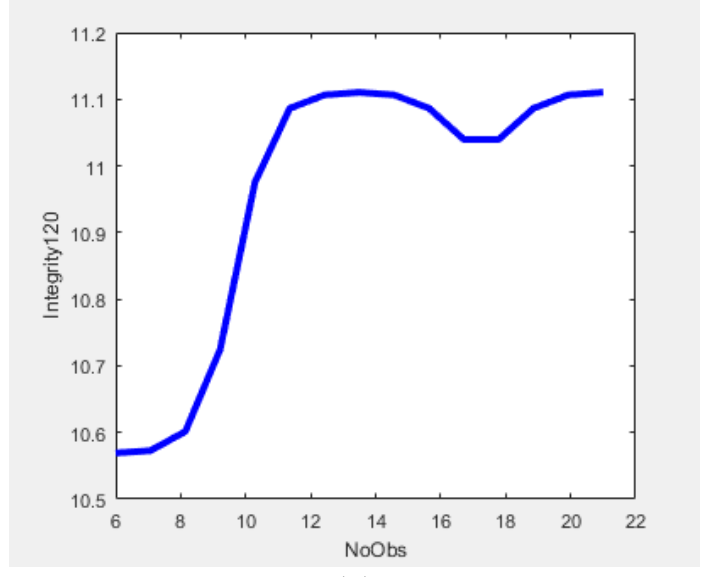

(a)

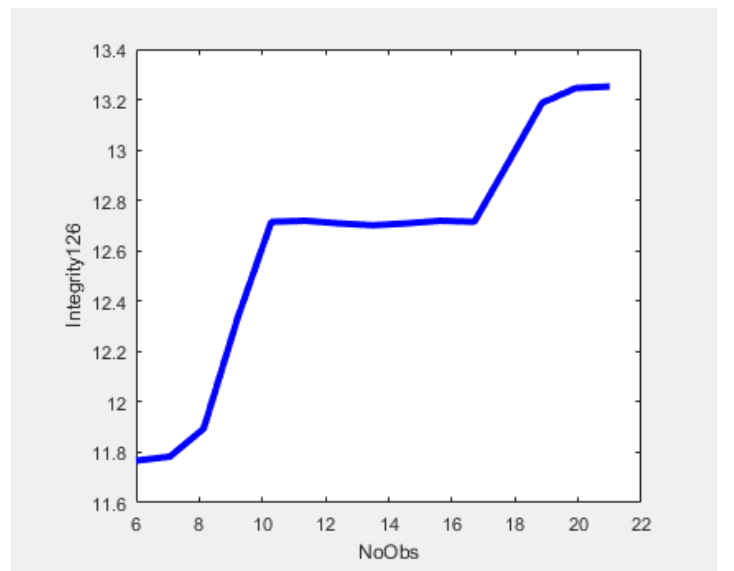

(b)

Figure 5. The correlation of the number of SSN observations $\left(x_{3}\right)$ and the integrity of PRN120 (a) and PRN126 (b) (Own study in MATLAB Fuzzy Logic Toolbox).

It can be also observed that changes in the standard deviation of SSN measurement correlate with the signal integrity (Figure 6). Of course, this comes from obvious correlation between the number of measurements carried out, and the related standard deviation of the measured parameter.

More interesting results come from SSN analysis. The correlation between the number of sunspots and signal integrity for both EGNOS satellites is presented in Figure 7. Interestingly, the signal in the analyzed period obtained the highest integrity when the solar activity, measured by the number of sunspots, reached an average value in the range of 101-160. It is also interesting that low SSN is correlated with low signal integrity. However, low solar activity is connected with more frequent occurrence of the coronal holes, which, in turn, are sources of high-speed solar wind that affect Earth's magnetosphere [30]. And this results in adverse ionospheric effects that influence signal integrity. On the other hand, high SSN is connected with a high TEC level and greater chances for intense geomagnetic storms, which, clearly, have negative effects [22]. This shows that SSN is not necessarily the best metric to be used in the context of GNSS signal integrity. Therefore, our future studies will include analysis of more directly related factors, such as TEC and geomagnetic indices. 


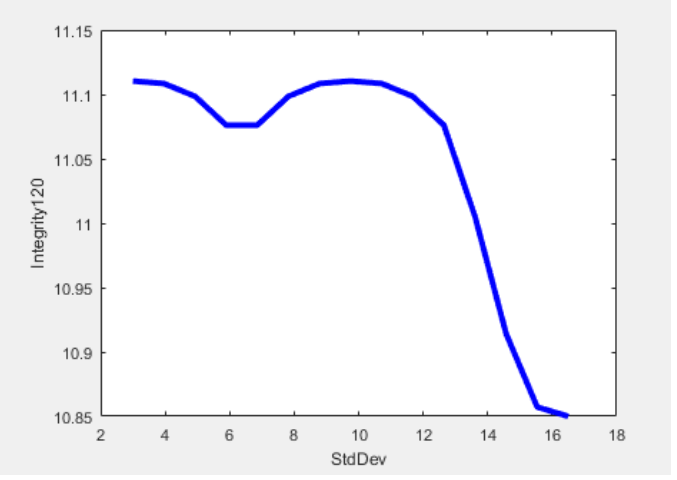

(a)

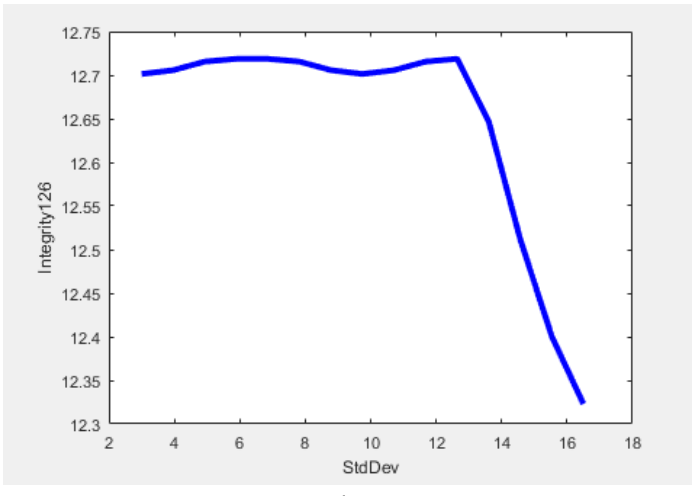

(b)

Figure 6. The SSN standard deviation $\left(x_{2}\right)$ and the integrity of PRN120 (a) and PRN126 (b) (Own study in MATLAB Fuzzy Logic Toolbox).

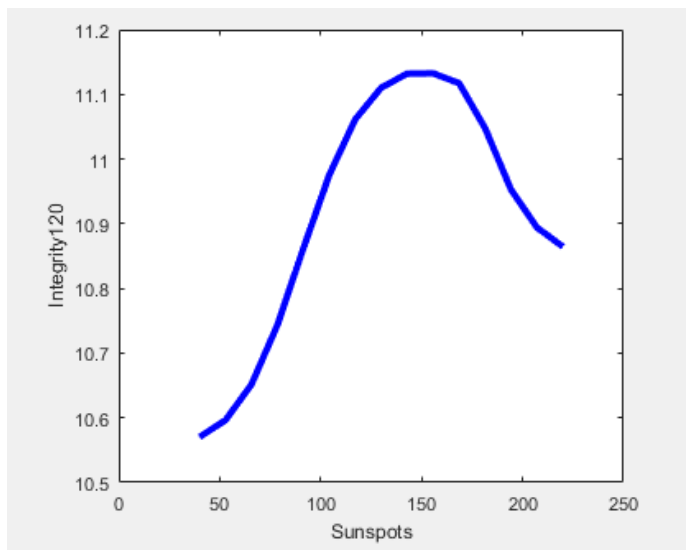

(a)

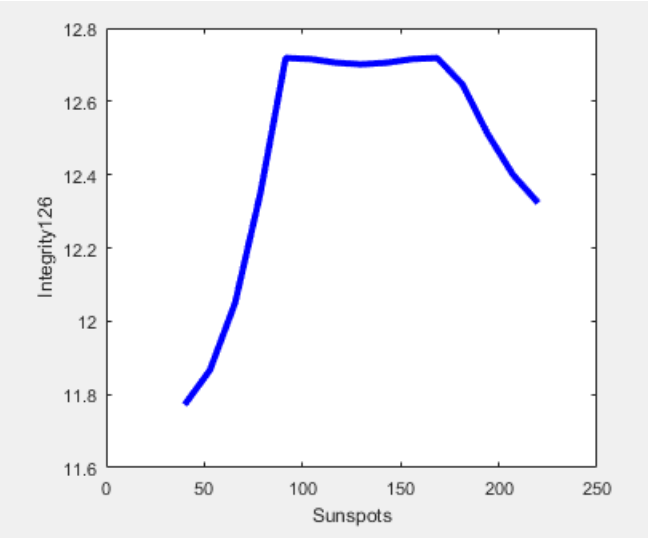

(b)

Figure 7. The influence of the sunspots $\left(x_{1}\right)$ on the integrity of PRN120 (a) and PRN126 (b) (Own study in MATLAB Fuzzy Logic Toolbox).

The last stage of the research is the process of defuzzification, i.e., searching for the non-fuzzy values of the variables that give the best result (highest integrity)-Figure 8 . The combination from Figure 8 was used as an example for the first 20 rules. A relatively high integrity value was achieved under certain input conditions: $x_{1}=127, x_{2}=9.88, x_{3}=20.6$.

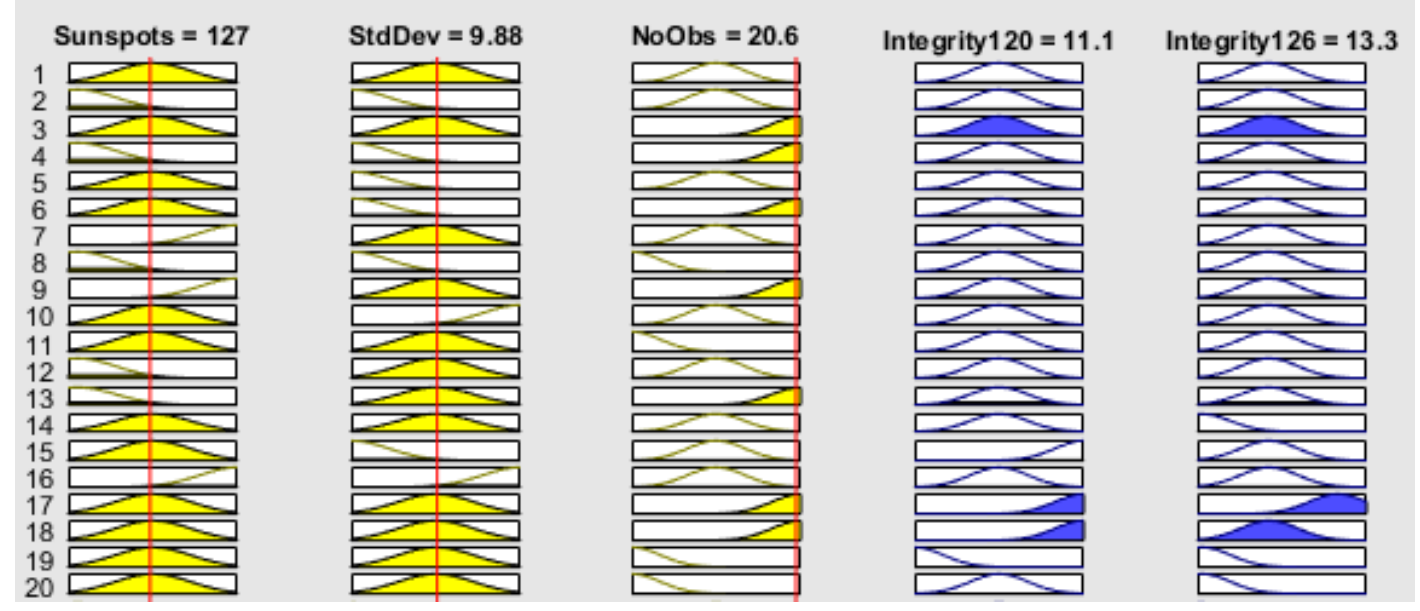

Figure 8. Graphical interpretation of the model defuzzification (Own study in MATLAB Fuzzy Logic Toolbox). 
The observations of behavior of the fuzzy model led to interesting conclusions, resulting from the research-the highest integrity of the satellite signal was observed for the "medium" value of the number of sunspots (in the range of 101-160). There is also surprisingly high correlation between measurements of SSN (number and std) and the signal integrity.

\section{Hellwigs' Method Verification and Results}

\subsection{Materials and Method}

The linguistic fuzzy model presented in the previous point must be verified. This verification is necessary to confirm, in a documented manner, that the models actually lead to the presented results. Moreover, this stage is particularly important when the studied phenomenon, and especially the research results, may have an impact on the safety of navigation of people, vehicles or robots. At the same time, in order not to generalize the results, the authors proposed a statistical verification of the direct influence of solar activity (defined by the number of sunspots) on the integrity of the satellite signal against the background of other atmospheric factors. Five variables were selected: cloudiness, relative air humidity, precipitation, temperature and atmospheric pressure.

The verification was performed using Hellwig's integral capacity method, also known as the optimal predicant selection method [52]. This is a formal technique for selecting the best explanatory variables. This technique is considered to be optimal because, as a result of verification, explanatory (input) variables with the largest impact on the explained (output) variable may be determined. Furthermore, the rules of Hellwig's verification method are as follows:

- it allows to eliminate the explanatory variables that do not affect the explained variable;

- $\quad$ explanatory (input) variables should be weakly related to each other, which prevents duplication of information provided by them;

- a large difference between the number of observations and the number of explanatory variables positively affects the accuracy of the predicate selection.

At the source of Hellwig's method lies the mathematical statistics and the correlation calculus. Its essence and, at the same time, the result reflect in a combination of explanatory variables where the integral information capacity is the largest. The entire calculation procedure can be summarized in a few steps:

1. the concept of the information carrier, which is the explanatory variable (input variable), is introduced;

2. the number of all possible combinations of explanatory variables is determined according to the formula:

$$
K=2^{m}-1
$$

where $m$ is the number of explanatory variables;

3. all types of combinations between explanatory variables are determined;

4. the individual capacity of information $h_{k j}$ is calculated according to the formula:

$$
h_{k j}=\frac{r_{j}^{2}}{1+\sum_{i \neq j}\left|r_{i j}\right|}
$$

where:

$k$-in $h$ is the number of combinations $k=1,2, \ldots, K$;

$j$-in $h$ specifying the number of the explanatory variables distinguished in a given combination remaining in dependencies with the explained variable;

$r_{j}$ - correlation coefficient of the $j$-th explanatory variable with the explained variable;

$r_{i j}$ - correlation coefficient of $i$-th and $j$-th explanatory variable $(i, j=1,2, \ldots, m)$; 
5. the integral capacity of the information carrier, $H_{k}$, for individual combinations of variables according to the formula is calculated:

$$
H_{k}=\sum_{j=1}^{n} h_{k j}
$$

6. the optimal combination of explanatory variables $H_{k}$ opt. - the one for which the integral information capacity is the largest is selected:

$$
H_{\text {kopt. }}=\max _{k} H_{k}
$$

For the purposes of calculations, the values of new variables were obtained in the tested time-series. The summary in Table 2 presents a measure of each variable and range of measurement values from the given period and station.

Table 2. Definition of explanatory (input) and explained (output) variables and their measure.

\begin{tabular}{cccc}
\hline $\begin{array}{c}\text { Input/ } \\
\text { Output }\end{array}$ & Variable & Measure & $\begin{array}{c}\text { The Range of Measurement } \\
\text { Values from a Given Period }\end{array}$ \\
\hline$x_{1}$ & Cloudiness & the degree of sky cover-age (on an octane scale) & $0-8$ \\
$x_{2}$ & Air humidity & in \% & $42-100$ \\
$x_{3}$ & Precipitation & measured mm of water column & $0-25$ \\
$x_{4}$ & Temperature & average air temperature in ${ }^{\circ} \mathrm{C}$ & $(-15)-24$ \\
$x_{5}$ & Atmospheric pressure & measured in hPa & $985-1024$ \\
$x_{6}$ & Solar activity & Number of sunspots & $40-220$ \\
$y$ & Integrity PRN120/PRN126 & PL-PE & $9.54-15.875$ \\
\hline
\end{tabular}

It is worth emphasizing that the selection of these variables for comparison was not accidental. In the literature, the role of the tropospheric factor in shaping the satellite signal path is repeatedly emphasized. For example, the tropospheric water vapor content is a derivative parameter from zenith wet delay (ZWD) and can be calculated as a function of the refractive index, depending on temperature, pressure and relative humidity [25-27]. Selbesoglu [24] claims that the troposphere affects GNSS signals due to the fast variability of the refraction index. Determination of the wet delay is more difficult than the hydrostatic delay due to much faster changes in the water vapor in the troposphere.

\subsection{Results and Discussion}

As presented in Table 2, the following explanatory variables will take part in the calculations:

- $\quad x_{1}$-cloudiness;

- $x_{2}$-air humidity;

- $x_{3}$-precipitation;

- $x_{4}$-temperature;

- $x_{5}$-atmospheric pressure;

- $x_{6}$ - solar activity.

The explained variables will be an integrated integrity from the satellite signal $(y)$. Therefore, the number of possible combinations of explained variables according to the Formula (8) is:

$$
K=2^{6}-1=64-1=63
$$

All types of combinations between explanatory variables are shown in Table 3 .

It is possible to calculate the individual capacities of the $h_{k j}$ information carrier after prior determination of correlation coefficients. Firstly, the correlation coefficients $r_{j}, j$-th explanatory variable with the explained variable. Secondly, the coefficients of correlation $r_{i j}, i$-th and $j$-th explanatory variable. The coefficient determining the level of linear 
dependence between random variables is Pearson's linear correlation. It accepts values between $[-1,1]$ and is expressed as the quotient of the covariance and the product of deviations of standard variables.

$$
r_{X Y}=\frac{\operatorname{cov}(X, Y)}{\sigma_{X} \sigma_{Y}}
$$

where:

$\sigma_{X}$-standard deviation $X$,

$\sigma_{Y}$-standard deviation $Y$.

Table 3. Combinations of explanatory variables.

\begin{tabular}{|c|c|c|}
\hline$K_{1}=\left\{x_{1}\right\}$ & $K_{22}=\left\{x_{1}, x_{2}, x_{3}\right\}$ & $K_{43}=\left\{x_{1}, x_{2}, x_{3}, x_{5}\right\}$ \\
\hline$K_{2}=\left\{x_{2}\right\}$ & $K_{23}=\left\{x_{1}, x_{2}, x_{4}\right\}$ & $K_{44}=\left\{x_{1}, x_{2}, x_{3}, x_{6}\right\}$ \\
\hline$K_{3}=\left\{x_{3}\right\}$ & $K_{24}=\left\{x_{1}, x_{2}, x_{5}\right\}$ & $K_{45}=\left\{x_{1}, x_{2}, x_{4}, x_{5}\right\}$ \\
\hline$K_{4}=\left\{x_{4}\right\}$ & $K_{25}=\left\{x_{1}, x_{2}, x_{6}\right\}$ & $K_{46}=\left\{x_{1}, x_{2}, x_{4}, x_{6}\right\}$ \\
\hline$K_{5}=\left\{x_{5}\right\}$ & $K_{26}=\left\{x_{1}, x_{3}, x_{4}\right\}$ & $K_{47}=\left\{x_{1}, x_{2}, x_{5}, x_{6}\right\}$ \\
\hline$K_{6}=\left\{x_{6}\right\}$ & $K_{27}=\left\{x_{1}, x_{3}, x_{5}\right\}$ & $K_{48}=\left\{x_{1}, x_{3}, x_{4}, x_{5}\right\}$ \\
\hline$K_{7}=\left\{x_{1}, x_{2}\right\}$ & $K_{28}=\left\{x_{1}, x_{3}, x_{6}\right\}$ & $K_{49}=\left\{x_{1}, x_{3}, x_{4}, x_{6}\right\}$ \\
\hline$K_{8}=\left\{x_{1}, x_{3}\right\}$ & $K_{29}=\left\{x_{1}, x_{4}, x_{5}\right\}$ & $K_{50}=\left\{x_{1}, x_{3}, x_{5}, x_{6}\right\}$ \\
\hline$K_{9}=\left\{x_{1}, x_{4}\right\}$ & $K_{30}=\left\{x_{1}, x_{4}, x_{6}\right\}$ & $K_{51}=\left\{x_{1}, x_{4}, x_{5}, x_{6}\right\}$ \\
\hline$K_{10}=\left\{x_{1}, x_{5}\right\}$ & $K_{31}=\left\{x_{1}, x_{5}, x_{6}\right\}$ & $K_{52}=\left\{x_{2}, x_{3}, x_{4}, x_{5}\right\}$ \\
\hline$K_{11}=\left\{x_{1}, x_{6}\right\}$ & $K_{32}=\left\{x_{2}, x_{3}, x_{4}\right\}$ & $K_{53}=\left\{x_{2}, x_{3}, x_{4}, x_{6}\right\}$ \\
\hline$K_{12}=\left\{x_{2}, x_{3}\right\}$ & $K_{33}=\left\{x_{2}, x_{3}, x_{5}\right\}$ & $K_{54}=\left\{x_{2}, x_{3}, x_{5}, x_{6}\right\}$ \\
\hline$K_{13}=\left\{x_{2}, x_{4}\right\}$ & $K_{34}=\left\{x_{2}, x_{3}, x_{6}\right\}$ & $K_{55}=\left\{x_{2}, x_{4}, x_{5}, x_{6}\right\}$ \\
\hline$K_{14}=\left\{x_{2}, x_{5}\right\}$ & $K_{35}=\left\{x_{2}, x_{4}, x_{5}\right\}$ & $K_{56}=\left\{x_{3}, x_{4}, x_{5}, x_{6}\right\}$ \\
\hline$K_{15}=\left\{x_{2}, x_{6}\right\}$ & $K_{36}=\left\{x_{2}, x_{4}, x_{6}\right\}$ & $K_{57}=\left\{x_{1}, x_{2}, x_{3}, x_{4}, x_{5}\right\}$ \\
\hline$K_{16}=\left\{x_{3}, x_{4}\right\}$ & $K_{37}=\left\{x_{2}, x_{5}, x_{6}\right\}$ & $K_{58}=\left\{x_{1}, x_{2}, x_{3}, x_{4}, x_{6}\right\}$ \\
\hline$K_{17}=\left\{x_{3}, x_{5}\right\}$ & $K_{38}=\left\{x_{3}, x_{4}, x_{5}\right\}$ & $K_{59}=\left\{x_{1}, x_{2}, x_{3}, x_{5}, x_{6}\right\}$ \\
\hline$K_{18}=\left\{x_{3}, x_{6}\right\}$ & $K_{39}=\left\{x_{3}, x_{4}, x_{6}\right\}$ & $K_{60}=\left\{x_{1}, x_{2}, x_{4}, x_{5}, x_{6}\right\}$ \\
\hline$K_{19}=\left\{x_{4}, x_{5}\right\}$ & $K_{40}=\left\{x_{3}, x_{5}, x_{6}\right\}$ & $K_{61}=\left\{x_{1}, x_{3}, x_{4}, x_{5}, x_{6}\right\}$ \\
\hline$K_{20}=\left\{x_{4}, x_{6}\right\}$ & $K_{41}=\left\{x_{4}, x_{5}, x_{6}\right\}$ & $K_{62}=\left\{x_{2}, x_{3}, x_{4}, x_{5}, x_{6}\right\}$ \\
\hline$K_{21}=\left\{x_{5}, x_{6}\right\}$ & $K_{42}=\left\{x_{1}, x_{2}, x_{3}, x_{4}\right\}$ & $K_{63}=\left\{x_{1}, x_{2}, x_{3}, x_{4}, x_{5}, x_{6}\right\}$ \\
\hline
\end{tabular}

Specialists [53,54] have interpreted its range, but it should not be treated too closely. For the needs of these studies, the below assumptions were taken. In this, if $r \neq 0$, variables are generally described as correlated. And moreover:

- if $0<|r| \leq 0.2$ this dependence is very low;

- if $0.2<|r| \leq 0.4$ this dependence is clear but low;

- if $0.4<|r| \leq 0.7$ this dependence is moderate;

- if $0.7<|r| \leq 0.9$ this dependence is significant;

- if $|r|>0.9$ this dependence is very strong;

- if $r=1$ or $r=-1$ this relationship occurs in the form of a linear function;

- if $r=0$ these variables are not correlated.

The absolute value of the correlation coefficient indicates the strength of statistical interdependence of variables, understood as a degree of determination of one variable value by the value of the second variable. It is worth mentioning that when the regression lines have a positive slope, this is unambiguous with a positive correlation of variables. On the other hand, when the regression lines have a negative slope, this indicates that the variables are negatively correlated and the correlation coefficient takes a negative value. At the same time, it should be noted that:

$$
r_{x_{1} x_{2}}=r_{x_{2} x_{1}}
$$

and the other coefficients can be considered in the same way. In Table 4 comparison of correlation coefficients for $r_{j}$ (explanatory variable with the explained variable) is shown. 
Table 4. Comparison of correlation coefficients values $r_{j}$.

\begin{tabular}{cccccc}
\hline $\mathbf{r}_{\mathbf{Y X} \mathbf{1}}$ & $\mathbf{r}_{\mathbf{Y X} \mathbf{2}}$ & $\mathbf{r}_{\mathbf{Y X} \mathbf{3}}$ & $\mathbf{r}_{\mathbf{Y X} \mathbf{4}}$ & $\mathbf{r}_{\mathbf{Y X 5}}$ & $\mathbf{r}_{\mathbf{Y X} \mathbf{6}}$ \\
\hline-0.17 & -0.01 & -0.27 & -0.12 & 0.16 & 0.24 \\
\hline
\end{tabular}

In Table 5 comparison of correlation coefficients for $r_{i j}(i$-th and $j$-th explanatory variable) is shown.

Table 5. Comparison of correlation coefficients values $r_{j}$.

\begin{tabular}{|c|c|c|c|c|}
\hline $\mathbf{r X 1 X 2}$ & $\mathbf{r}_{X 1 X 3}$ & $\mathbf{r}_{X 1 X 4}$ & rX1X5 & $\mathbf{r}_{X 1 \times 6}$ \\
\hline 0.64 & 0.30 & -0.28 & -0.43 & -0.08 \\
\hline $\mathbf{r}_{\mathbf{X} 2 X 3}$ & $\mathbf{r}_{X 2 X 4}$ & $\mathbf{r}_{\mathbf{X} 2 X 5}$ & $\mathbf{r}_{X 2 X 6}$ & \\
\hline 0.32 & -0.39 & -0.32 & 0.09 & \\
\hline $\mathbf{r}_{\mathbf{X} 3 \mathbf{X}}$ & $\mathbf{r}_{\mathbf{X} 3 \mathbf{5}}$ & $\mathbf{r}_{\mathbf{X} 3 \times 6}$ & & \\
\hline 0.15 & -0.24 & -0.14 & & \\
\hline $\mathbf{r}_{X 4 X 5}$ & $\mathbf{r}_{\mathbf{X} 46}$ & & & \\
\hline-0.11 & -0.17 & & & \\
\hline $\mathbf{r}_{\mathbf{X} 5 \times 6}$ & & & & \\
\hline 0.02 & & & & \\
\hline
\end{tabular}

The next stage of Hellwig's method is the calculation of the capacity of the individual information carrier $h_{k j}$. A large number of combinations between explanatory variables generates a complex process of determining all individual capacities. For order, the calculation example below shows the individual capacities of the two selected combinations $K_{17}, K_{53}$ :

$$
\begin{gathered}
K_{17}=\left\{x_{3}, x_{5}\right\} \\
K_{53}=\left\{x_{2}, x_{3}, x_{4}, x_{6}\right\} \\
h_{173}=\frac{r_{y_{1} x_{3}}^{2}}{1+\left|r_{x_{3} x_{5}}\right|}=0.059 \\
h_{175}=\frac{r_{y_{1} x_{5}}^{2}}{1+\left|r_{x_{3} x_{5}}\right|}=0.021 \\
h_{532}=\frac{r_{y_{1} x_{2}}^{2}}{1+\left|r_{x_{2} x_{3}}\right|+\left|r_{x_{2} x_{4}}\right|+\left|r_{x_{2} x_{6}}\right|}=0.0 \\
h_{533}=\frac{r_{y_{1} x_{3}}^{2}}{1+\left|r_{x_{2} x_{3}}\right|+\left|r_{x_{3} x_{4}}\right|+\left|r_{x_{3} x_{6}}\right|}=0.045 \\
h_{534}=\frac{r_{y_{1} x_{4}}^{2}}{1+\left|r_{x_{2} x_{4}}\right|+\left|r_{x_{3} x_{4}}\right|+\left|r_{x_{4} x_{6}}\right|}=0.008 \\
h_{536}=\frac{r_{y_{1} x_{6}}^{2}}{1+\left|r_{x_{2} x_{6}}\right|+\left|r_{x_{3} x_{6}}\right|+\left|r_{x_{4} x_{6}}\right|}=0.041
\end{gathered}
$$

The fifth step of the presented method is to calculate the integral capacities of the information carrier $H_{k}$ for individual combinations of variables. For previously selected combinations, the formula for determining the value of $H_{k}$ is presented below. This formula refers to the combination of $K_{17}$ and takes the form:

$$
H_{17}=h_{173}+h_{175}=0.079
$$


And for $K_{53}$ presents as follows:

$$
H_{53}=h_{532}+h_{533}+h_{534}+h_{536}=0.095
$$

The last, but the key, step of the method is to select the optimal combination of explanatory variables $H_{k}$ opt. . The list of $H_{k}$ opt. values for each combination is shown in Table 6.

Table 6. The list of $H_{k}$ opt. values for each combination.

\begin{tabular}{cccccc}
\hline No. of $\boldsymbol{K}$ & $\boldsymbol{H}_{\boldsymbol{k} \text { opt. }}$ & No. of $\boldsymbol{K}$ & $\boldsymbol{H}_{\boldsymbol{k} \text { opt. }}$ & $\boldsymbol{N o . ~ o f ~} \boldsymbol{K}$ & $\boldsymbol{H}_{\boldsymbol{k} \text { opt. }}$ \\
\hline$K_{1}$ & 0.029 & $K_{22}$ & 0.060 & $K_{43}$ & 0.064 \\
$K_{2}$ & 0.000 & $K_{23}$ & 0.024 & $K_{44}$ & 0.100 \\
$K_{3}$ & 0.073 & $K_{24}$ & 0.029 & $K_{45}$ & 0.034 \\
$K_{4}$ & 0.014 & $K_{25}$ & 0.066 & $K_{46}$ & 0.065 \\
$K_{5}$ & 0.026 & $K_{26}$ & 0.079 & $K_{47}$ & 0.076 \\
$K_{6}$ & 0.058 & $K_{27}$ & 0.080 & $K_{48}$ & 0.081 \\
$K_{7}$ & 0.018 & $K_{28}$ & 0.119 & $K_{49}$. & 0.114 \\
$K_{8}$ & 0.079 & $K_{29}$ & 0.044 & $K_{50}$ & 0.121 \\
$K_{9}$ & 0.034 & $K_{30}$ & 0.077 & $K_{51}$ & 0.086 \\
$K_{10}$ & 0.038 & $K_{31}$ & 0.089 & $K_{52}$ & 0.067 \\
$K_{11}$ & 0.080 & $K_{32}$ & 0.059 & $K_{53}$ & 0.095 \\
$K_{12}$ & 0.056 & $K_{33}$ & 0.063 & $K_{54}$ & 0.105 \\
$K_{13}$ & 0.010 & $K_{34}$ & 0.097 & $K_{55}$ & 0.071 \\
$K_{14}$ & 0.019 & $K_{35}$ & 0.028 & $K_{56}$ & 0.120 \\
$K_{15}$ & 0.053 & $K_{36}$ & 0.055 & $K_{57}$ & 0.067 \\
$K_{16}$ & 0.076 & $K_{37}$ & 0.071 & $K_{58}$ & 0.097 \\
$K_{17}$ & 0.079 & $K_{38}$ & 0.083 & $K_{59}$ & 0.104 \\
$K_{18}$ & 0.114 & $K_{39}$ & 0.112 & $K_{60}$ & 0.075 \\
$K_{19}$ & 0.036 & $K_{40}$ & 0.123 & $K_{61}$ & 0.117 \\
$K_{20}$ & 0.062 & $K_{41}$ & 0.082 & $K_{62}$ & 0.103 \\
$K_{21}$ & 0.082 & $K_{42}$ & 0.062 & $K_{63}$ & 0.102 \\
\hline
\end{tabular}

And, as a result of the calculations carried out, the maximum value of $H_{k \text { opt. }}$ was determined for $K_{40}$ (signed with yellow in Table 6).

$$
H_{\text {opt. }}=0.123
$$

The largest value of the integral information capacity was related to the combination $K_{40}$, in which $x_{3}, x_{5}, x_{6}$ explanatory variables performed. Variable $x_{3}$ corresponds to the precipitation, while $x_{5}$ corresponds to pressure. Variable $x_{6}$ is responsible for solar activity. This suggests that even though the wet delay is relatively small $(10-20 \mathrm{~cm})$, its fast changes associated with passages of weather-fronts may have a negative effect on signal integrity.

\section{Conclusions}

The purpose of this article was to attempt to quantify the effect of solar activity (expressed by sunspots) and tropospheric parameters on the satellite navigation signal integrity using fuzzy logic. Due to the fuzzy model, the highest integrity of the satellite signal was observed for the "medium" value of the number of sunspots (in the range 101-160). Integrity seemed to be very sensitive to changes in solar activity manifested by a significant decrease or a significant increase in the number of sunspots. What is more, attention was also paid to other atmospheric (tropospheric) conditions and, in this case, it was verified whether, against the background of cloudiness, precipitation, humidity, pressure and temperature, solar activity affects the integrity to the greatest extent. Interestingly, despite the fact that the tropospheric water vapor content (which is a derivative parameter from ZWD) and its components are considered in the context of the impact on the GNSS signal, it was actually the precipitation which played an important 
role in these studies. This is the same as solar activity. These observations resulted from the statistical analysis and led to the following conclusions:

1. some influence of the solar activity on the integrity of the satellite signal was confirmed, despite the addition of five other tropospheric factors to the study;

2. statistical analysis showed that precipitation was influencing the integrity of the satellite signal as much as SSN;

3. the values of individual information carriers for solar activity and precipitation were very close ( 0.050 and 0.053 , respectively);

4. of the three factors generally having the greatest impact on satellite signal integrity, atmospheric pressure recorded the lowest value;

5. the remaining three factors (cloudiness, air humidity and temperature) in the analyzed period had the least impact on the explained variable. Precipitation is less frequently described in the literature as a factor affecting the satellite signal. According to stateof-the-art research [55-57], such a factor is air humidity and, often, temperature. In practice, these two factors have a negligible effect on signal integrity. Note that this study applies to the EGNOS system and European mid-latitudes only, and the results for other SBAS systems and areas might be different.

Due to the authors' opinion that the SSN together with precipitation may influence integrity the most, these parameters are considered interesting for further research. Therefore, our future studies will focus on statistical analysis of the influence of other space-weather phenomena having more direct impact on GNSS signal integrity.

Author Contributions: Conceptualization, K.K.-P.; E.D.; P.W.; B.M.; J.M.B.; methodology, K.K.-P.; P.W.; B.M.; software, K.K.-P.; validation, E.D.; P.W.; B.M.; formal analysis, K.K.-P.; E.D.; resources, K.K.-P.; writing—original draft preparation, K.K.-P.; E.D.; P.W.; B.M.; J.M.B.; writing-review and editing, E.D.; J.M.B.; supervision, P.W.; project administration, K.K.-P.; funding acquisition, K.K.-P. All authors have read and agreed to the published version of the manuscript.

Funding: Research was funded by the Centre for Priority Research Area Artificial Intelligence and Robotics of Warsaw University of Technology within the Excellence Initiative: Research University (IDUB) programme (Contract No. 1820/29/Z01/POB2/2021).

Institutional Review Board Statement: Not applicable.

Informed Consent Statement: Not applicable.

Conflicts of Interest: The authors declare no conflict of interest. The funders had no role in the design of the study; in the collection, analyses, or interpretation of data; in the writing of the manuscript, or in the decision to publish the results.

\section{References}

1. Hofmann-Wellenhof, B.; Lichtenegger, H.; Wasle, E. GNSS-Global Navigation Satellite Systems: GPS, GLONASS, Galileo, and More; Springer Science \& Business Media: New York, NY, USA, 2007.

2. Krzykowska, K.; Krzykowski, M. Forecasting Parameters of Satellite Navigation Signal through Artificial Neural Networks for the Purpose of Civil Aviation. Int. J. Aerosp. Eng. 2019, 2019, 7632958. [CrossRef]

3. Liu, J.; Rizos, C.; Cai, B. A Hybrid Integrity Monitoring Method Using Vehicular Wireless Communication in Difficult Environments for GNSS. Veh. Commun. 2020, 23, 100229. [CrossRef]

4. Emmens, T.; Amrit, C.; Abdi, A.; Ghosh, M. The Promises and Perils of Automatic Identification System Data. Expert Syst. Appl. 2021, 178, 114975. [CrossRef]

5. Ogaja, C.; Rizos, C.; Han, S. Is GPS Good Enough for Monitoring the Dynamics of High-Rise Buildings. In Proceedings of the 2nd Trans Tasman Surveyors Congress, Queenstown, New Zealand, 20-26 August 2000.

6. Zhu, Z.; Li, C. Study on Real-Time Identification of GNSS Multipath Errors and Its Application. Aerosp. Sci. Technol. 2016, 52, 215-223. [CrossRef]

7. Liu, H.; Xu, L.; Shu, B.; Zhang, M.; Qian, C. A New Method to Improve the Performance of Multi-GNSS Pseudorange Positioning in Signal-Degraded Environment. Adv. Space Res. 2016, 58, 577-586. [CrossRef]

8. Galán, C.O.; Pérez, J.R.; Cortés, S.G.; Sánchez, A.B. Analysis of the Influence of Forestry Environments on the Accuracy of GPS Measurements by Means of Recurrent Neural Networks. Math. Comput. Model. 2013, 57, 2016-2023. [CrossRef] 
9. Wielgosz, P. Quality Assessment of GPS Rapid Static Positioning with Weighted Ionospheric Parameters in Generalized Least Squares. Gps Solut. 2011, 15, 89-99. [CrossRef]

10. Goss, A.; Hernández-Pajares, M.; Schmidt, M.; Roma-Dollase, D.; Erdogan, E.; Seitz, F. High-Resolution Ionosphere Corrections for Single-Frequency Positioning. Remote Sens. 2021, 13, 12. [CrossRef]

11. Tomaszewski, D.; Wielgosz, P.; Rapiński, J.; Krypiak-Gregorczyk, A.; Kaźmierczak, R.; Hernández-Pajares, M.; Yang, H.; OrúsPérez, R. Assessment of Centre National d'Etudes Spatiales Real-Time Ionosphere Maps in Instantaneous Precise Real-Time Kinematic Positioning over Medium and Long Baselines. Sensors 2020, 20, 2293. [CrossRef]

12. Hoque, M.M.; Jakowski, N.; Berdermann, J. Positioning Performance of the NTCM Model Driven by GPS Klobuchar Model Parameters. J. Space Weather Space Clim. 2018, 8, A20. [CrossRef]

13. Hoque, M.M.; Jakowski, N.; Orús-Pérez, R. Fast Ionospheric Correction Using Galileo Az Coefficients and the NTCM Model. Gps Solut. 2019, 23, 1-12. [CrossRef]

14. Hernández-Pajares, M.; Juan, J.M.; Sanz, J.; Colombo, O.L. Application of Ionospheric Tomography to Real-Time GPS CarrierPhase Ambiguities Resolution, at Scales of 400-1000 Km and with High Geomagnetic Activity. Geophys. Res. Lett. 2000, 27, 2009-2012. [CrossRef]

15. Aguilera, C.; Lorenzo, J.M. EGNOS in Aviation: Strategy and Implementation Status. In Proceedings of the EGNOS Service Provision Workshop, Athens, Greece, 3-4 October 2017.

16. Ciećko, A. Analysis of the EGNOS Quality Parameters during High Ionosphere Activity. IET RadarSonar Navig. 2019, 13, 1131-1139. [CrossRef]

17. Oliveira, J.; Tiberius, C. Quality Control in SBAS: Protection Levels and Reliability Levels. J. Navig. 2009, 62, 509. [CrossRef]

18. Bijjahalli, S.; Ramasamy, S.; Sabatini, R. A GNSS Integrity Augmentation System for Ground Vehicle Operations. Energy Procedia 2017, 110, 149-155. [CrossRef]

19. Flament, D.; Thomas, D.; Lopez, C.; Melinotte, J.-M.; Urbanska, K.; Derambure, X.; Boisseau, A. EGNOS System Evolutions in Europe and within the International Multi-SBAS Context. In Proceedings of the 2020 European Navigation Conference (ENC), Dresden, Germany, 23-24 November 2020; pp. 1-13.

20. Xu, H.; Wang, J.; Zhan, X. GNSS Satellite Autonomous Integrity Monitoring (SAIM) Using Inter-Satellite Measurements. Adv. Space Res. 2011, 47, 1116-1126. [CrossRef]

21. Yang, Y.; Xu, J. GNSS Receiver Autonomous Integrity Monitoring (RAIM) Algorithm Based on Robust Estimation. Geod. Geodyn. 2016, 7, 117-123. [CrossRef]

22. Ciećko, A.; Grunwald, G. Klobuchar, NeQuick G, and EGNOS Ionospheric Models for GPS/EGNOS Single-Frequency Positioning under 6-12 September 2017 Space Weather Events. Appl. Sci. 2020, 10, 1553. [CrossRef]

23. Ibanez Segura, D.; Rovira Garcia, A.; Alonso, M.T.; Sanz, J.; Juan, J.M.; Gonzalez Casado, G.; López Martínez, M. EGNOS 1046 Maritime Service Assessment. Sensors 2020, 20, 276. [CrossRef] [PubMed]

24. Selbesoglu, M.O. Prediction of Tropospheric Wet Delay by an Artificial Neural Network Model Based on Meteorological and GNSS Data. Eng. Sci. Technol. Int. J. 2020, 23, 967-972. [CrossRef]

25. Mohammed, J. Artificial Neural Network for Predicting Global Sub-Daily Tropospheric Wet Delay. J. Atmos. Sol. Terr. Phys. 2021, 217, 105612. [CrossRef]

26. Wielgosz, P.; Krukowska, M.; Paziewski, J.; Krypiak-Gregorczyk, A.; Stepniak, K.; Kaplon, J.; Sierny, J.; Hadas, T.; Bosy, J. Performance of ZTD Models Derived in near Real-Time from GBAS and Meteorological Data in GPS Fast-Static Positioning. Meas. Sci. Technol. 2013, 24, 125802. [CrossRef]

27. Sun, Z.; Zhang, B.; Yao, Y. Improving the Estimation of Weighted Mean Temperature in China Using Machine Learning Methods. Remote Sens. 2021, 13, 1016. [CrossRef]

28. Hoque, M.; Jakowski, N.; Berdermann, J. Transionospheric Microwave Propagation: Higher-Order Effects up to 100 GHz; Intech Open Science Open Minds: London, UK, 2017; pp. 15-38.

29. Leick, A.; Rapoport, L.; Tatarnikov, D. GPS Satellite Surveying; John Wiley \& Sons: Hoboken, NJ, USA, 2015.

30. Hunsucker, R.D.; Hargreaves, J.K. The High-Latitude Ionosphere and Its Effects on Radio Propagation; Cambridge University Press: Cambridge, UK, 2007.

31. Yin, P.; Mitchell, C.N. Demonstration of the Use of the Doppler Orbitography and Radio Positioning Integrated by Satellite (DORIS) Measurements to Validate GPS Ionospheric Imaging. Adv. Space Res. 2011, 48, 500-506. [CrossRef]

32. Habarulema, J.B.; McKinnell, L.-A.; Opperman, B.D. Towards a GPS-Based TEC Prediction Model for Southern Africa with Feed Forward Networks. Adv. Space Res. 2009, 44, 82-92. [CrossRef]

33. Sur, D.; Paul, A. Comparison of Standard TEC Models with a Neural Network Based TEC Model Using Multistation GPS TEC around the Northern Crest of Equatorial Ionization Anomaly in the Indian Longitude Sector during the Low and Moderate Solar Activity Levels of the 24th Solar Cycle. Adv. Space Res. 2013, 52, 810-820. [CrossRef]

34. Krypiak-Gregorczyk, A.; Wielgosz, P. Carrier Phase Bias Estimation of Geometry-Free Linear Combination of GNSS Signals for Ionospheric TEC Modeling. GPS Solut. 2018, 22, 1-9. [CrossRef]

35. Schreiner, W.S.; Weiss, J.P.; Anthes, R.A.; Braun, J.; Chu, V.; Fong, J.; Hunt, D.; Kuo, Y.-H.; Meehan, T.; Serafino, W. COSMIC-2 Radio Occultation Constellation: First Results. Geophys. Res. Lett. 2020, 47, e2019GL086841. [CrossRef]

36. Chen, S.-Y.; Liu, C.-Y.; Huang, C.-Y.; Hsu, S.-C.; Li, H.-W.; Lin, P.-H.; Cheng, J.-P.; Huang, C.-Y. An Analysis Study of FORMOSAT7/COSMIC-2 Radio Occultation Data in the Troposphere. Remote Sens. 2021, 13, 717. [CrossRef] 
37. Sunspot Index and Long-Term Solar Observations. Available online: https://wwwbis.sidc.be/silso/archiveyearlyssnplot (accessed on 20 March 2021).

38. Krzykowska, K.; Dudek, E. How to Prevent Loss of Satellite Navigation Continuity Caused by Nimbostratus? Concept for Polish ATM. Transp. Res. Procedia 2019, 43, 63-73. [CrossRef]

39. Weber, L. International Civil Aviation Organization; Kluwer Law International BV: Alphen aan den Rijn, The Netherlands, 2017.

40. Siergiejczyk, M.; Krzykowska, K.; Rosinski, A. Evaluation of the influence of atmospheric conditions on the quality of satellite signal. In Marine Navigation; CRC Press: Boca Raton, FL, USA, 2017; pp. 121-128.

41. Krzykowska, K.; Siergiejczyk, M.; Rosiński, A. Influence of selected external factors on satellite navigation signal quality. In Safety and Reliability-Safe Societies in a Changing World; CRC Press: Boca Raton, FL, USA, 2018; pp. 701-705.

42. Siergiejczyk, M.; Rosiński, A.; Krzykowska, K. Reliability assessment of supporting satellite system EGNOS. In New Results in Dependability and Computer Systems; Springer: Berlin/Heidelberg, Germany, 2013; pp. 353-363.

43. Tossaint, M.; Samson, J.; Toran, F.; Ventura-Traveset, J.; Hernández-Pajares, M.; Juan, J.M.; Sanz, J.; Ramos-Bosch, P. The Stanford-ESA Integrity Diagram: A New Tool for the User Domain Sbas Integrity Assessment. Navigation 2007, 54, 153-162. [CrossRef]

44. European Space Agency. EGNOS Fact Sheet. 2021. Available online: www.egnos-pro.esa.int (accessed on 20 March 2021).

45. Zadeh, L.A. Fuzzy Sets. Inf. Control 1965, 8, 338-353. [CrossRef]

46. Gharajeh, M.S.; Jond, H.B. Hybrid Global Positioning System-Adaptive Neuro-Fuzzy Inference System Based Autonomous Mobile Robot Navigation. Robot. Auton. Syst. 2020, 134, 103669. [CrossRef]

47. Kumar, P.B.; Muni, M.K.; Parhi, D.R. Navigational Analysis of Multiple Humanoids Using a Hybrid Regression-Fuzzy Logic Control Approach in Complex Terrains. Appl. Soft Comput. 2020, 89, 106088. [CrossRef]

48. Kaleta, W.; Skorupski, J. A Fuzzy Inference Approach to Analysis of LPV-200 Procedures Influence on Air Traffic Safety. Transp. Res. Part C Emerg. Technol. 2019, 106, 264-280. [CrossRef]

49. Grzesik, N. Fuzzy Sets in Aircraft System Efficiency Evaluation. Aircr. Eng. Aerosp. Technol. 2016. [CrossRef]

50. Wang, C.Y. Topological Characterizations of Generalized Fuzzy Rough Sets. Fuzzy Sets Syst. 2017, 312, 109-125. [CrossRef]

51. Yazdanbakhsh, O.; Dick, S. A Systematic Review of Complex Fuzzy Sets and Logic. Fuzzy Sets Syst. 2018, 338, 1-22. [CrossRef]

52. Duda, A. Hellwig Method as a Quantitative Method Selection of Variables for Modeling Logistic Processes. Gospod. Mater. I Logistyka 2018, 11, 75-108.

53. Akoglu, H. User's Guide to Correlation Coefficients. Turk. J. Emerg. Med. 2018, 18, 91-93. [CrossRef]

54. Xiang, J.X. Estimation of Minimum and Maximum Correlation Coefficients. Stat. Probab. Lett. 2019, 145, 81-88. [CrossRef]

55. Xia, P.; Xia, J.; Ye, S.; Xu, C. A New Method for Estimating Tropospheric Zenith Wet-Component Delay of GNSS Signals from Surface Meteorology Data. Remote Sens. 2020, 12, 3497. [CrossRef]

56. Zhu, B.; Li, J.; Tang, W. Correcting InSAR Topographically Correlated Tropospheric Delays Using a Power Law Model Based on ERA-Interim Reanalysis. Remote Sens. 2017, 9, 765. [CrossRef]

57. Selbesoglu, M.O. Spatial Interpolation of GNSS Troposphere Wet Delay by a Newly Designed Artificial Neural Network Model. Appl. Sci. 2019, 9, 4688. [CrossRef] 